\title{
La recepción de un texto de Heinrich Böll por lectores mexicanos*
}

\section{Ute SEYDEL}

\author{
Una anécdota para bajar los ánimos de trabajar ${ }^{1}$
}

En un puerto de la costa occidental de Europa un hombre, con vestimenta pobre, está acostado, dormitando, en su lancha de pescar. Un turista de traje elegante está poniendo un rollo de color en su cámara para sacar fotos de la imagen idilica: el cielo azul, el mar verde con crestas de olas pacíficas y blancas, la lancha negra, el gorro rojo del pescador, clic. Otra vez clic y, por si las dudas, una tercera vez: clic. El ruido seco, casi hostil, despierta al pescador que estaba dormitando y que se levanta soñoliento, y soñoliento pesca su cajetilla de cigarrillos; pero antes de encontrar lo buscado, el turista, presto, ya le está ofreciendo uno. No es que se lo hubiera puesto en la boca, pero sí se lo puso en la mano y un cuarto clic, el del mechero, termina la cortesía apresurada.

Por este exceso de cortesía precipitada, apenas mensurable, que tampoco se puede comprobar, ha surgido una situación embarazosa y tensa que trata de conciliar el turista — que sí domina el idioma del lugar-mediante una conversación.

- Hará una pesca buena.

El pescador niega con la cabeza.

- Pero me han dicho que el tiempo está favorable.

El pescador afirma con la cabeza.

- ¿O sea que no va a salir a pescar?

El pescador niega con la cabeza; el turista se pone cada vez más nervioso.

- El presente artículo está basado en un estudio de mayor envergadura, que se llevó a cabo de 1985 a 1988 con el titulo Deutsche Literatur in den Bildungsinstitutiones Mexikos. En ese trabajo se explica más detalladamente tanto la manera de proceder en el curso de literatura, los criterios para elegir los textos, etcétera, como la recepción del relato de $\mathrm{H}$. Böll por los estudiantes mexicanos. (Ute SEYDEL, Deutsche Literatur in den Bildungsinstitutionen Mexikos, pp. 120-141.)

' La siguiente traducción fue elaborada por mí con ayuda de la doctora Elisabeth Siefer, del Departamento de Letras Alemanas de la Facultad de Filosofia y Letras de la UNAM. 
Cierto, a él le importa el bienestar del hombre vestido modestamente; cierto, la tristeza de la oportunidad perdida le duele y acongoja.

$-\measuredangle \mathrm{O}$ es que usted no se siente bien?

Finalmente, el pescador abandona el lenguaje de los gestos para llegar a la palabra realmente hablada.

- Me siento de perlas - dice-, jamás me sentí mejor.

Se levanta, se estira, como si quisiera exhibir su cuerpo atlético.

- Me siento muy bien.

La expresión de la cara del turista se vuelve cada vez más infeliz. Ya no puede reprimir la pregunta que, por decirlo así, amenaza con romperle el corazón.

- ¿Pero por qué no sale luego?

La respuesta viene de inmediato y es precisa:

- Porque ya he salido esta mañana.

$-¿$ Y fue buena la pesca?

-Fue tan buena que no hace falta que salga de nuevo. Tengo cuatro langostas en mis cèstas y he capturado dos docenas de macarelas...

El pescador, finalmente despierto, sale de su reserva y da unas palmaditas en el hombro del turista, como para tranquilizarlo. La expresión de aquél le parece de una pena que, si bien está fuera de lugar, sí conmueve.

- Hasta tengo suficiente para mañana y pasado mañana - dice, para aliviar el alma del forastero. - ¿Desea uno de mis cigarrillos?

-Sí, gracias.

Ambos se colocan los cigarrillos en la boca, un quinto clic. El forastero, meneando la cabeza, se sienta sobre el borde de la lancha, pone a un lado la cámara, porque ahora necesita las dos manos para darle énfasis a su discurso.

- No es que quiera meterme en sus asuntos, pero imagínese nada más que usted saliera hoy una segunda vez, tal vez una tercera y hasta una cuarta y que capturara tres, cuatro, cinco y tal vez hasta seis docenas de macarelas, imagínese nada más.

El pescador afirma con la cabeza.

-Usted podría - sigue el turista - no solamente hoy, no; también mañana, pasado mañana, ¿qué le digo?, tal vez cada dia, cuando hace buen tiempo, salir dos o tres veces, $y$ tal vez hasta cuatro veces; ¿sabe qué pasaría entonces?

El pescador niega con la cabeza.

- A más tardar en un año, podría comprarse un motor; en dos años, una segunda lancha; en tres o cuatro años tal vez podría tener un pequeño bote. Con las dos lanchas y con el bote pescaria mucho más en un día, claro está. Tendría dos botes, haría... -el entusiasmo le corta la voz por un momento - construiría un pequeño frigorífico, tal vez una ahumadora; más tarde una procesadora de escabeche... daría 
vueltas en un helicóptero, podría divisar los cardúmenes de peces desde arriba y dar indicaciones por radio a sus botes. Usted podría adquirir los derechos de pesca para el salmón, abrir un restaurante de pescado, exportar directamente a París la langosta, sin intermediario, y luego... - de nuevo el entusiasmo hace enmudecer al forastero. Meneando la cabeza, triste en lo más profundo de su corazón, ya casi perdiendo su alegría vacacional, está mirando la marea que se acerca apaciblemente $y$ en donde dan brincos alegremente los peces no pescados.

-Y luego --dice, y de nuevo la emoción le hace imposible hablar. El pescador le da palmaditas en el hombro, como a un niño que se atragantó.

- ¿Y luego qué? - pregunta en voz baja.

- Luego - dice el forastero con quieto entusiasmo-, luego puede estar sentado aquí en el puerto con toda tranquilidad, estar dormitando en el sol y mirar este maravilloso mar.

-Pero esto lo estoy haciendo ya ahora - replica el pescador-, estoy tranquilo en el puerto dormitando. Sólo los clics de su cámara me han molestado en esto.

De hecho el turista, instruido de tal manera, se retiró pensativo, pues siempre habia creido que estaba trabajando para ya no tener que hacerlo un día, y ya no tenía ni una pizca de compasión con el pescador modestamente vestido, sólo un poco de envidia. ${ }^{2}$

¿Cómo reaccionarán los lectores de distintos ámbitos culturales a este texto? En eso consistía mi interés al aplicar la teoría de la recepción a un caso concreto.

Los pasos del proceder de la teoría de la recepción al tratar un texto literario se reflejan en la estructura del presente artículo. Primero se resume y analiza el texto literario en su estructura, tomando en cuenta sus "vacíos". ${ }^{3}$

Esta interpretación se basa en las ya hechas por investigadores literarios alemanes, quienes toman en cuenta la reacción al texto por lectores de la República Federal de Alemania, que lo leyeron en el momento de su aparición.

\footnotetext{
${ }^{2}$ Texto en alemán: “Anekdote zur Senkung der Arbeitsmoral”, en Heinrich Böll Werke, Romane und Erzählungen. 4. 1961-1970, pp. 267-269.

${ }^{3}$ W. Iser crea el término Leerstelle (W. ISER, “Die Appellstruktur der Texte”, en R. WARNING, ed., Rezeptionsästhetik, pp. 228 y 252). En la antología de D. Rall, este término está vertido al español por la traductora Sandra Franco como "vacio" (W. ISER, "La estructura apelativa de los textos", en D. RALL, ed., En busca del texto. Teorla de la recepción literaria, p. 106).
} 
Asimismo, se considera la "relevancia representativa" del texto para los coetáneos alemanes. ${ }^{4}$ Por el concepto de "relevancia representativa" se entiende lo pertinente que fue el texto en el momento de su creción en la sociedad de Alemania Occidental. Se investiga hasta qué punto el tema y los aspectos formales del texto han sido típicos de ese momento.

Después se hace una hipótesis sobre la relevancia del texto para los destinatarios concretos, que en la mayoría de los casos lo leen con una diferencia de tiempo y, a veces, ante un fondo cultural distinto. Debido a esto, se analiza el horizonte de recepción, que depende de los trasfondos culturales y sociales de los lectores concretos. De ahi se deduce una hipótesis sobre el interés de los lectores concretos, en este caso de los estudiantes mexicanos de mi curso de literatura, ante el texto. De esta manera se pueden anticipar posibles reacciones.

El artículo se basa esencialmente en un "diálogo entre lectores" que se llevó a cabo con estudiantes mexicanos que asistían a las clases de alemán en el Centro de Enseñanza de Lenguas Extranjeras de la UNAM en 1985. La última parte de este artículo esboza el transcurso de una clase, en concreto, y evalúa las diferencias entre la hipótesis sobre la reacción de los estudiantes mexicanos y su respuesta real después de haber leído el texto.

El relato se leyó en un curso de literatura en el cual participaron estudiantes de distintas disciplinas. Sus conocimientos del alemán correspondían al "Certificado Alemán como Lengua Extranjera" (Zertifikat Deutsch als Fremdsprache). Los estudiantes casi no tenían conocimientos previos sobre literatura alemana.

\section{El texto}

\section{Tema y estructura del texto}

En el relato se desarrolla un diálogo entre un turista alemán y un pescador que es ciudadano de algún país del oeste de Europa.

Al llegar a la playa, el turista se queda impresionado por el panorama pintoresco. Saca varias fotos y los ruidos de la cámara despiertan al pescador.

${ }^{4}$ F. HEBEL, “Die Rolle der Literatur in der Kulturvermittlung oder: Gibt es eine 'repräsentative Relevanz' von Texten? Erörtert am Beispiel von Gerhard Hauptmanns Komödie 'Der Biberpelz" ", en A. WIERLACHER, ed., Fremdsprache Deutsch 2, pp. 387-388.

${ }^{5}$ D. KRUSCHE, “Lese-Unterschiede.Zum interkulturellen Leser-Gespräch”, en Jahrbuch Deutsch als Fremdsprache 7, p. 1. 
El hecho de que el pescador no esté pescando, a pesar de haber un clima ideal para hacerlo, asombra e inquieta al turista. El que la razón para descansar no sea una enfermedad, sino el hecho de ya tener suficientes peces, asombra al visitante. Trata de convencer al pescador de que salga a pescar nuevamente y que, en general, cambie su disposición ante el trabajo. Desarrolla un plan para la posible expansión económica del pescador, que al mismo tiempo lo sacaría de la miseria. La meta lejana, después de una vida dedicada al trabajo, sería, según el turista, una vida tranquila sin preocupaciones económicas. El pescador responde lacónicamente que ya se siente plenamente realizado. En el epílogo el narrador comenta que ante el argumento del pescador, el turista ya no siente compasión sino, más bien, envidia.

El texto se puede dividir en cuatro partes: primera, la introducción al escenario; segunda, la primera parte del diálogo, en la cual contrasta la abundancia de signos verbales del turista con la escasez de signos verbales del pescador; tercera, la segunda parte del diálogo, en la cual domina el monólogo del turista y, por fin, la cuarta parte, constituida por el epílogo, con el clímax y el comentario del narrador. El comentario contiene la moraleja del texto y le otorga su dimensión didáctica. En oposición a la mayor parte del relato, que está escrito en presente, el epílogo está en imperfecto.

\section{La representatividad temática del texto}

El texto de H. Böll aparece en el año 1963, cuando la política de restauración del canciller $\mathrm{K}$. Adenauer había llegado a su punto culminante. En la década de los cincuentas la República Federal de Alemania logró ser internacionalmente reconocida y una buena perspectiva para los negocios, gracias a un inesperado crecimiento económico, el llamado Wirtschaftswunder.

En esa época, muchos escritores criticaron de manera creciente el fanatismo por el alto rendimiento en la producción y la fe en el progreso de capas amplias de la sociedad. Protestaron contra la opinión difundida de que resistirse al progreso significaba actuar contra el raciocinio economicista. ${ }^{6}$ De esta manera, los escritores se anticiparon, hasta cierto punto, al pesimismo ante el progreso que se manifestó, más tarde, en las décadas de los setentas y ochentas en los movimientos ecologistas.

${ }^{6}$ M. DURZAK, Die deutsche Kurzgeschichte der Gegenwart. Autorenporträts, Werkstattgespräche, Interpretationen, p. 385. 
En otro cuento H. Böll llevó al absurdo la planeación racional e industrial. Mientras que en ese texto, un cuento corto titulado "La estación de Zimpren", 7 contrasta la planeación económica con la razón ecológica de una agricultura, en la "Anécdota" traslada la confrontación de dos formas de vida al extranjero, se puede suponer que a una región pobre del suroeste de Europa. De esa manera, se permite criticar al alemán viajero como representante del insensible turismo masivo, que ni siquiera en sus vacaciones logra tranquilizarse ni relajarse. Asimismo, se critica el afán civilizador de muchos miembros de las naciones ricas del norte de Europa. ${ }^{8}$

El clímax es unánimemente interpretado por la crítica literaria alemana como el surgimiento de una alternativa de vida más digna que el estilo de vivir de muchos alemanes, casi exclusivamente orientado hacia metas económicas.

Estos textos de H. Böll, entre otros, incluyendo el texto leído con los estudiantes mexicanios, se distinguen no sólo por el tema, que es típico de los años sesentas, sino también por la forma de representación satírico-irónica que se utilizó frecuentemente en ese tiempo.

\section{Los destinatarios concretos}

\section{Hipótesis sobre la relevancia del texto en México}

"Una anécdota para bajar los ánimos de trabajar" es un texto intercultural, en el cual se encuentran dos individuos. Sólo los rasgos característicos de la cultura de uno de los interlocutores (a saber, de la alemana) quedan definidos. El origen cultural del otro personaje, el pescador, no se define. Sólo se le ubica socialmente. Por eso el contraste cultural que se presenta no es específico. En cambio, se ve un país equis a través de la perspectiva de un turista alemán. Para el trabajo en el salón de clase, esto tiene la ventaja de que los estudiantes pueden aceptar 0 rechazar, más libremente, la oferta del texto de indentificarse con uno de los dos personajes, sin sentirse forzados a defender comportamientos determinados de un miembro de su cultura.

${ }^{7}$ Heinrich BơLL, "Der Bahnhof von Zimpren", en Heinrich Böll Werke, Romane und Erzählungen. 3. 1958-1959, pp. 282-292.

${ }^{8} \mathrm{~K}$.ZOBEL, "Heinrich Böll: Anekdote zur Senkung der Atbeitsmoral", en Textanalysen. Eine Einfuhrung in die Interpretation moderner Kurzprosa, p. 183. 
El tema del texto, la problematización de los patrones de pensamiento y acción de miembros de una sociedad industrializada moderna, me pareció interesante para la lectura con los estudiantes mexicanos. Supuse que la crítica de un escritor de la República Federal Alemana a esa forma de vida llevaría a una discusión interesante, dado que ésa se presenta, muchas veces, como modelo a los países del llamado "Tercer Mundo". Además, presumi que el texto daría un buen motivo para la discusión sobre el turismo moderno y el afán civilizador de muchos viajeros del llamado "Primer Mundo".

No sólo me decidí en favor de este texto por su representatividad temática y su relevancia para los estudiantes, sino también por su estructura. Por el contraste entre dos maneras de pensar y actuar y por el clímax, seguido por la moraleja del texto, éste me parecía apropiado para su didactización -es decir, su preparación para ser utilizado con fines didácticos. También la apertura del texto, respecto a la posible identificación con uno de los dos personajes, ponderaba en su favor.

Consideré como difícil únicamente el estilo satírico-irónico, porque los lectores que tienen el alemán como lengua extanjera no lo captan tan fácilmente.

\section{El horizonte de recepción de los estudiantes mexicanos}

La cultura mexicana es, como puede deducirse de trabajos científicos o ensayos de distintos filósofos, sociólogos y escritores, una cultura heterogénea, en la cual coexisten, en variantes múltiples, concepciones mágico-indígenas, católico-españolas y occidentales-modernas. En algunos casos también se encuentran en conflicto mutuo.

La brevedad de este artículo sólo permite dar una caracterización y una subdivisión a grandes rasgos. Para la representación más detallada de esta cuestión quisiera remitir a los trabajos de Olien, Paz y Ramos. ${ }^{9}$

Con base en las distintas concepciones del tiempo, se puede dividir la sociedad en dos grupos principales. Es posible distinguir entre el grupo en el cual prevalece una concepción del tiempo circular y el otro, en el que domina una concepción lineal. El primer grupo abarca las capas marginadas, de pocos recursos, que se caracterizan por patrones de pensamiento indígenas y en las cuales se reproducen formas de compor-

\footnotetext{
${ }^{9}$ M. OLIEN, Latin Americans-Contemporary Peoples and their Cultural Traditions; O. PAZ, Tiempo nublado, El laberinto de la soledad y Los signos en rotación yotros ensayos; S. RAMOS, El perfil del hombre y la cultura en México. Véase también U. SEYDEL, op. cit., pp. $48-54$ y 129-133.
} 
tamiento y de producción. Sus miembros viven en los barrios pobres de las ciudades o en el campo como peones. Octavio Paz califica a esa cultura como inmóvil. Según él, dominan la "inercia" y el fatalismo. ${ }^{10}$ Además, tanto la relación de los hombres entre sí como la de los hombres con la naturaleza están marcadas por la violencia. " Con esta cultura, caracterizada por las tradiciones autóctonas, contrasta una cultura urbana que se orienta hacia modelos europeos.

La concepción lineal del tiempo está relacionada con la herencia cristiano-occidental. Ella implica cierto optimismo frente al progreso, pero más moderado del que se tiene en las culturas europeas. Al constituir la parte activa de la población, los miembros de este grupo tratan de imponer su modelo de vida a los grupos sociales que consideran como subdesarrollados.

En ambos grupos la imagen de la naturaleza varía mucho de aquella que se tiene en el norte de Europa. La naturaleza no representa un mundo idílico, sino hostil y amenazante. 0 . Paz describe la relación que se tiene en México con la naturaleza de la siguiente forma: "En el valle de México el hombre se siente suspendido entre el cielo y la tierra y oscila entre fuerzas contrarias, [...] el mundo que nos rodea existe por sí mismo, tiene vida propia y no ha sido inventado, como en los Estados Unidos, por el hombre". 12

Tomando en cuenta estas consideraciones, se podía suponer que los estudiantes trasladarían el contraste entre ambas culturas a la propia y que no considerarían que la cultura del pescador debiera ser necesariamente valorada en forma positiva por estar vinculada a la naturaleza y pertenercer a un mundo opuesto al del turista.

\section{Transcurso de la clase y evaluación de los resultados obtenidos}

\section{Transcurso de la clase}

Antes de leer el texto no proporcioné información acerca de la vida del autor, ni acerca de las circunstancias en las cuales se escribió el relato; solamente se repartió éste con un glosario.

${ }^{10}$ O. PAZ, "El pachuco y otros extremos", en El laberinto de la soledad, p. 11.

$" \mathrm{~A}$. DORFMANN ("La violencia en la novela latinoamericana actual", en Imaginación $y$ violencia en América, pp. 9-42) trata de explicar las causas de la violencia en muchos países latinoamericanos, que también se refleja en numerosas novelas latinoamericanas.

12 O. PAZ, "El pachuco y otros extremos", en El laberinto de la soledad, p. 18. 
Por los comentarios de los estudiantes, se podía llegar a la conclusión de que su primera comprensión del texto se basó en las partes dialogadas. No captaron la exageración satírica, en la caracterización del turista, en las partes narrativas. Acerca del clímax, comentaron que no sintieron sinceros los sentimientos de envidia que muestra el turista. ${ }^{13}$

En cambio, desarrollaron un panorama de la vida mexicana en el campo, la inseguridad social, el alcoholismo, el deficiente servicio médico, la violencia y también las escasas posibilidades de ascenso social. Este se ve obstaculizado por la dependencia de comerciantes intermediarios, que sólo compran cierta cantidad de pescado; además, por problemas de transporte, por la dificultad de conseguir créditos, etcétera. Por eso los proyectos que hace el turista con la vida del pescador no son realizables desde el punto de vista de los estudiantes mexicanos. Una reacción interesante fue que no sólo criticaron al turista por su afán civilizador y por la poca comprensión de la situación del pescador, sino que también criticaron al pescador. Expusieron que de igual manera el pescador, con su parquedad, no contribuía a que el turista entendiera mejor sus condiciones de vida.

Los estudiantes en su critica hasta reprocharon a $\mathrm{H}$. Böll el tener una perspectiva parcial, porque presentaba la vida del pescador como un mundo mejor y deseable, sin hacer comentarios o reflexiones críticas. ${ }^{14}$

Sólo cuando se hubo terminado el análisis exacto del texto, en el cual se vio tanto la manera en que se guía al lector para ver a los personajes desde distintas perspectivas, como el uso de los tiempos y modos verbales, en especial el uso del subjuntivo (Konjunktiv II) en las partes monólogas, tuvieron que modificar su crítica inicial.

Finalmente, pudieron captar por qué la intención de $\mathrm{H}$. Böll de criticar a la sociedad competitiva hizo que describiera el mundo del pescador sólo a grandes rasgos, sin entrar en detalles o cuestionarlo, para que, así, sirviera como mundo contrastante.

Digno de mencionar es, además, que los estudiantes no querian entrar en el segundo nivel de la crítica, el que se refiere al turismo de masas.

\section{Evaluación}

El transcurso de la clase mostró que un diálogo entre los lectores, que arranca de la primera comprensión (primäres Verstehen) ${ }^{15}$ de los estu-

${ }^{13}$ U. SEYDEL, op. cit., pp. 134-135.

14 Ibid., p. 134.

is F. HERMANNS, "Doppeltes Verstehen. Überlegungen zur Begrūndung einer 
diantes, puede llevar a una discusión interesante sobre las distintas concepciones respecto a la naturaleza y a los diferentes conceptos de desarrollo. Además, hizo evidente que el profesor de literatura alemana como literatura de una cultura ajena, tiene que poseer conocimientos bien fundados sobre la cultura de los estudiantes, para así poder interpretar los malentendidos que son de indole cultural y aprovecharlo como motivo para la discusión. ${ }^{16}$

En el diálogo entre lectores efectuado con los estudiantes mexicanos se podrían abarcar de forma contrastada temas como la vida en el campo, las concepciones sobre la naturaleza y los conceptos para el desarrollo de la sociedad.

Sólo cuando el texto sigue siendo el centro de interés en el diálogo entre lectores, el proceso de la lectura y de su comprensión pueden convertirse en un "acto ejemplar de formación de experiencia" ( $A k t$ exemplarischer Erfahrungsbildung). ${ }^{17}$

\section{Bibliografía}

BACHMANN-MEDICK, D., "Kulturelle Texte und interkulturelles (MißVerstehen. Kulturanthropologische Herausforderungen für die interkulturelle Literaturwissenschaft)", en A. WIERLACHER, ed., Perspektiven und Verfahren interkultureller Germanistik. Beiträge der Bayreuther Tagung vom 1.-4.7.87. Múnich, 1987, pp. 653-664. (Beiträge der Gesellschaft für interkulturelle Germanistik, 3)

BöLL, Heinrich, "Der Bahnhof von Zimpren" (1958), en Heinrich Böll Werke, Romane und Erzählungen. 3. 1958-1959. Ed. de B. Balzer. Gütersloh, [s. a.,] pp. 282-292.

BöLL, Heinrich, "Anekdote zur Senkung der Arbeitsmoral”, en Heinrich Böll Werke, Romane und Erzählungen. 4. 1961-1970. Ed. de B. Balzer. Colonia, 1977, pp. 267-269.

dialogischen Hermeneutik", en A. WIERLACHER, ed., Perspektiven und Verfahren interkultureller Germanistik..., p. 149.

${ }^{16}$ Acerca de la importancia de hablar sobre "malentendidos" (MiB-Verstehen) en clases de literatura, ver las publicaciones de D. BACHMANN-MEDICK, "Kulturelle Texte und interkulturelles...", en A. WIERLACHER, ed., Perspektiven und Verfahren interkultureller Germanistik...; D. KRUSCHE, op. cit., y F. HERMANNS, op. cit.

${ }^{17}$ D. KRUSCHE, op. cit., p. 1. 
DORFMANN, Ariel, "La violencia en la novela latinoamericana actual", en Imaginación y violencia en América. 2a. ed. Barcelona, 1972, pp. 9-42.

DURZAK, M., Die deutsche Kurzgeschichte der Gegenwart. Autorenporträts, Werkstattgespräche, Interpretationen. Stuttgart, 1980.

HeBEL, F., "Die Rolle der Literatur in der Kulturvermittlung oder: Gibt es eine 'repräsentative Relevanz' von Texten? Erörtert am Beispiel von Gerhard Hauptmanns Komödie 'Der Biberpelz' ", en A. WIERLACHER, ed., Fremdsprache Deutsch 2. Múnich, 1980, pp. 387-410.

Hermanns, F., "Doppeltes Verstehen. Überlegungen zur Begründung einer dialogischen Hermeneutik", en A. WIERLACHER, ed., Perspektiven und Verfahren interkultureller Germanistik. Beiträge der Bayreuther Tagung vom 1.-4.7.87. Múnich, 1987, pp. 145-155. (Beiträge der Gesellschaft für interkulturelle Germanistik, 3)

ISER, W., "La estructura apelativa de los textos", en D. RALL, ed., En busca del texto. Teoría de la recepción literaria. Trad. de S. Franco et al. México, UNAM, 1987, pp. 99-121.

ISER, W., "Die Appellstruktur der Texte", en R. WARNING, ed., Rezeptionsästhetik. Múnich, pp. 228 y 252.

KRUSCHE, D., "Lese-Unterschiede. Zum interkulturellen Leser-Gespräch", en Jahrbuch Deutsch als Fremdsprache 7. 1981, pp. 1-17.

OLIEN, M., Latin Americans-Contemporary Peoples and their Cultural Traditions. NuevaYork, 1973.

PAZ, Octavio, Tiempo nublado. 2a. ed. México, 1983.

PAZ, Octavio, El laberinto de la soledad. 13a. ed. México, FCE, 1984.

PAZ, Octavio, Los signos en rotación y otros ensayos. 3a. ed. Pról. de Carlos Fuentes. Madrid, 1986.

Ramos, Samuel, El perfil del hombre y la cultura en México. 12a. ed. México, 1984. 
150 LA RECEPCIÓN DE UN TEXTO DE HEINRICH BÖLL

SEYDEL, Ute, Deutsche Literatur in den Bildungsinstitutionen Mexikos. Tesis, Ludwig-Maximilians-Universität. Múnich, 1988.

ZoBEL, K., "Heinrich Böll: Anekdote zur Senkung der Arbeitsmoral”, en Textanalysen. Eine Einführung in die Interpretation moderner Kurzprosa. Paderborn/Múnich/Viena/Zúrich, 1985, pp. 180-185. 